\title{
MODELOS DE MATURIDADE: DO CMM AOS MODELOS PARA INDÚSTRIA 4.0
}

Isaac Ambrosio da Silva (isaac.ambr@gmail.com) - Programa de Pós-Graduação em Sistemas Mecatrônicos - PPMEC, Universidade de Brasília (UnB)

Sanderson César Macêdo Barbalho (sandersoncesar@unb.br) - Programa de Pós-Graduação em Sistemas Mecatrônicos - PPMEC, Universidade de Brasília (UnB)

\section{RESUMO}

Diante do contexto da quarta revolução industrial, ou Indústria 4.0, novos conceitos são criados, tornando fábricas inteligentes e alterando a forma como os produtos são manufaturados e processos são planejados, agregando mais valor ao produto e ao cliente. Considerando o impacto das tecnologias emergentes sobre toda a cadeia produtiva, diversos modelos de maturidade, capabilidade ou prontidão surgem com o objetivo de avaliar em que ponto a organização se encontra e quais passos são necessários para a melhoria de seus processos. Com isso, este trabalho visa analisar a evolução dos modelos de maturidade nos últimos anos, através de uma revisão bibliométrica, desde o CMM até o modelo desenvolvido para Indústria 4.0 pela Acatech.

Palavras chave: Maturidade; Capabilidade; Indústria 4.0; Acatech, CMM 


\section{INTRODUÇÃ̃}

A Manufatura Avançada, ou Indústria 4.0, está intimamente relacionada com outros conceitos como a comunicação máquina a máquina (Gorecky el al, 2014), tecnologia de identificação por radiofrequência (RFID) (Wang, 2014), Sistemas Ciber-Físicos (CPS) (Lee, Bagheri e Kao, 2015), a Internet das coisas (IoT), a Internet de serviços (IoS), cloud computing (Drath e Horch, 2014), inteligência computacional (CI), data mining e sistemas de apoio a tomada de decisão (Li, Wang e Wang, 2017).

O termo Indústria 4.0 refere-se coletivamente a uma ampla gama de conceitos atuais, cuja classificação clara sobre uma disciplina, bem como sua distinção precisa, não é possível em casos individuais. Alguns dos conceitos fundamentais são: fábrica inteligente, sistemas cyber-físicos, auto-organização, adaptação às necessidades humanas, responsabilidade social corporativa e novos sistemas de distribuição, aquisição e serviços no desenvolvimento de produtos (Lazi et al, 2014).

Abordagens existentes na manufatura utilizando técnicas de mineração de dados podem ser divididas em cinco áreas de aplicação: análise de qualidade de produtos para correlacionar a saída qualidade e parâmetros do sistema; análise de falha de recursos de produção; análise de manutenção para melhorar a disponibilidade de recursos de produção; planejamento de produção e análise de cronograma para melhorar a qualidade do planejamento; e o planejamento estratégico e análise de cronograma para melhorar o relacionamento com o cliente e aumentar as vendas (Spendla et al, 2017).

$\mathrm{Na}$ literatura, existem diversos modelos de maturidade, padrões, metodologias e diretrizes que podem auxiliar uma organização a se tornar mais competitiva. Inspirado pelo movimento de garantia de qualidade do século XX, o SEI (Software Engineering Institute), Centro de Pesquisa da Carnegie Mellon University que opera com financiamento do Departamento de Defesa dos Estados Unidos, por solicitação do governo americano, começou, em 1986, a desenvolver um modelo de maturidade de processo e um questionário que ajudaria organizações a melhorar a qualidade dos seus processos de software: Capability Maturity Model for Software (CMM).

Com o advento da quarta revolução industrial, novos modelos de maturidade surgem, com o objetivo de avaliar as organizações em diversas áreas para o ingresso na chamada Indústria 4.0 e auxiliar nas melhorias necessárias. 
O trabalho aqui apresentado tem como objetivo avaliar os modelos de maturidade desenvolvidos nos últimos anos, com destaque para o Modelo de Maturidade e Capabilidade CMMI e para o Modelo de Maturidade para Indústria 4.0 da Acatech. Para tanto, uma pesquisa bibliométrica dos modelos de maturidade para Indústria 4.0 foi realizada a partir da base de dados SCOPUS.

Na seção seguinte define-se o conceito de indústria 4.0 e apresenta-se o modelo de maturidade CMM. Na terceira seção é apresentada uma pesquisa bibliométrica sobre os modelos de maturidade para Indústria $4.0 \mathrm{com}$ destaque para o modelo da Acatech. Por fim a conclusão é realizada e encerra-se com as referências bibliográficas utilizadas no texto.

\section{REVISÃO TEÓRICA}

\subsection{Indústria 4.0}

Ao longo dos últimos anos tem-se observado o avanço da utilização de novas tecnologias na cadeia produtiva em todo o mundo. Desde o início da industrialização, os processos produtivos sofrem influências e transformações através das tecnologias desenvolvidas, pelas demandas do mercado e também pelo surgimento de novos modelos de negócios (Melo, Cavalcanti e Gonçalves, 2007).

Recentemente, devido ao desenvolvimento dos sistemas de comunicação, da Internet e ao crescente melhoramento da microeletrônica, tornando os componentes menores e com maior processamento, aumentaram as pesquisas que buscam a comunicação, integração e uma maior dinamicidade da manufatura através de habilitadores como Internet das Coisas (IoT) e Sistemas Cyber Físicos (CPS).

A Quarta Revolução estabelece um novo paradigma para os sistemas de produção, compreendendo uma fábrica inteligente que opera de forma mais autônoma e com menor intervenção humana. Novos conceitos surgem diante desse cenário: Big Data, Cloud Computing, Inteligência Artificial, Internet Industrial das Coisas (IIoT), Segurança Cibernética (Lee, Bagheri e Kao, 2015).

O termo Indústria 4.0, referente à Quarta Revolução Industrial, derivado do termo alemão industrie 4.0, foi apresentado em 2011 na Feira de Hannover e surgiu como um projeto de alta tecnologia do ministério federal de educação e pesquisa do governo alemão, com o objetivo de aumentar a automatização dos sistemas produtivos locais (Jazdi, 2014). 


\subsection{Modelo CMM}

Observou-se que as organizações geralmente concentram seus esforços nas seguintes áreas: pessoas, procedimentos e métodos e, ferramentas e equipamentos (Figura 1). Sendo que o que mantém a harmonia entre estas três dimensões são os processos.

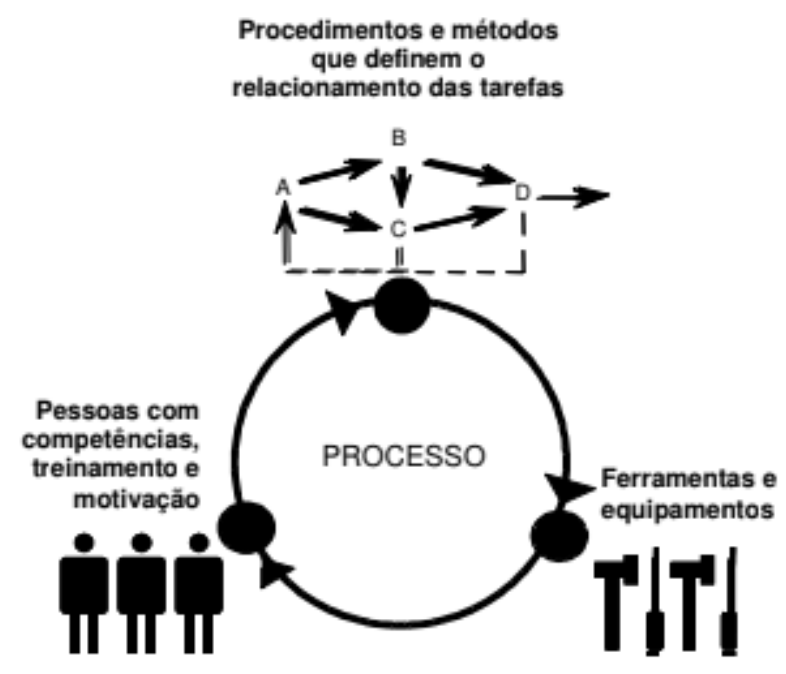

FIGURA 1. Dimensões críticas (CMMI, 2002)

Utilizando da premissa de que a qualidade de um sistema ou produto é altamente influenciada pelo processo utilizado para desenvolvê-lo e mantê-lo (Paulk, 2009), o SEI na década de 80 desenvolveu alguns CMMs, voltados para melhoria de processos em uma organização.

A partir de 1991 foram criados CMMs modelos voltados para: Engenharia de Sistemas, Engenharia de Software, Aquisição de Software, Gestão e Desenvolvimento de Força de Trabalho, e Desenvolvimento Integrado de Processo e Produto (IPPD). Observou-se que, apesar dos modelos se mostrarem úteis para diversas organizações, estes não eram integráveis e quando da utilização de vários modelos, tornara-se inviável do ponto de vista de treinamento, avaliações e atividades de melhoria.

Em 2000, com a finalidade de integrar os diversos modelos CMM, surge o CMMI (Capability Maturity Model Integration), modelo de maturidade que sugere o que deve ser aplicado aos processos de desenvolvimento integrado de hardware e software nas organizações e define 22 áreas de processos, organizados em quatro grupos: Gerenciamento de processos, Gerenciamento de projetos, Engenharia e Apoio (Helgesson, Höst e Weyns, 2012). 
Visando resolver o problema gerado da utilização de vários CMMs, iniciou-se o desenvolvimento de um framework único, o CMMI, combinando três modelos:

1. Capability Maturity Model for Software (SW-CMM)

2. Systems Engineering Capability Model (SECM)

3. Integrated Product Development Capability Maturity Model (IPD-CMM)

No modelo CMMI, é possível avaliar as melhorias em processos sob dois aspectos: contínuo, utilizando níveis de capacidade, e por estágios, caracterizado por níveis de maturidade.

A representação por estágios utiliza conjuntos predefinidos de áreas de processo para definir um caminho de melhoria para uma organização. Esse caminho de melhoria é caracterizado por níveis de maturidade. Cada nível de maturidade contém um conjunto de áreas de processos que caracterizam diferentes comportamentos organizacionais.

Posteriormente o CMMI foi organizado em três componentes: Desenvolvimento, Serviços e Aquisição:

1. Desenvolvimento: provê orientação para gerenciamento, avaliação e monitoramento dos processos em desenvolvimento;

2. Serviços: Provê orientação para prestação de serviços dentro das organizações e para consumidores externos;

3. Aquisição: Provê orientação para permitir liderança nas atividades de aquisição de produtos ou serviços de outros fornecedores.

Na Tabela 1 são apresentados os níveis de capabilidade e maturidade de acordo com o CMMI

TABELA 1. Níveis de Capabilidade e Maturidade segundo o CMMI

\begin{tabular}{|c|c|}
\hline Capabilidade & Maturidade \\
\hline $\begin{array}{c}\text { Capacidade do processo em atender } \\
\text { plenamente às especificações do Cliente ou } \\
\text { da Engenharia }\end{array}$ & $\begin{array}{c}\text { Relacionada à qualidade dos processos } \\
\text { utilizados em uma organização no } \\
\text { desenvolvimento e manutenção de um } \\
\text { determinado produto }\end{array}$ \\
\hline Nível 0: Incompleto & - \\
\hline
\end{tabular}




\begin{tabular}{|l|l|}
\hline Nível 1: Executado & Nível 1: Inicial \\
\hline Nível 2: Gerenciado & Nível 2: Gerenciado \\
\hline Nível 3: Definido & Nível 3: Definido \\
\hline Nível 4: Gerenciado Quantitativamente & Nível 4: Gerenciado Quantitativamente \\
\hline Nível 5: Em Otimização & Nível 5: Em Otimização \\
\hline
\end{tabular}

Os níveis de capabilidade, associados à representação contínua, aplicam-se à melhoria de processo da organização em áreas de processos individuais. Esses níveis são um meio para melhorar, de forma incremental, os processos correspondentes a uma determinada área de processo. Há seis níveis de capabilidade, numerados de 0 a 5. (CMMI, 2002).

Níveis de maturidade, associados à representação por estágios, aplicam-se à melhoria de processo da organização em um conjunto de áreas de processo. Esses níveis auxiliam na previsão dos resultados de futuros projetos. Há cinco níveis de maturidade, numerados de 1 a 5.

1. Inicial: representa processos caóticos, sem uma sequência pré-definida (ad-hoc), sendo que o sucesso depende exclusivamente da competência das pessoas.

2. Gerenciado: Os processos são planejados e executados de acordo com uma política. Em momentos de crise, a disciplina de processo contribui para que as práticas existentes sejam mantidas.

3. Definido: Os processos são bem caracterizados e entendidos, e são descritos em padrões, procedimentos, ferramentas e métodos, de forma mais rigorosa do que no nível anterior. Os processos geralmente são previsíveis apenas de forma qualitativa.

4. Gerenciado Quantitativamente: A organização e os projetos estabelecem objetivos quantitativos para qualidade e para desempenho de processo, utilizando-os como critérios na gestão de processos. O desempenho dos processos é controlado por meio de técnicas estatísticas e outras técnicas quantitativas.

5. Em Otimização: Foco na melhoria contínua do desempenho de processo por meio de melhorias incrementais e inovadoras de processo e de tecnologia. 
Organizações com baixos níveis de maturidade apresentam características de processos improvisados e com grande dependência das pessoas, acarretando o desconhecimento do progresso dos projetos, ocasionando custos excessivos de operação e manutenção. Em contraponto, organizações com altos graus de maturidade possuem processos bem definidos, documentados e continuamente aprimorados.

A capabilidade está relacionada a uma evolução da condição de atingir, cada vez mais, melhores resultados dos processos, sendo que esses são mantidos sob controle em quaisquer condições. O termo capabilidade se difere em seu contexto da palavra capacidade. Enquanto capacidade é relacionada com a quantidade a ser produzida, capabilidade é ter essa capacidade claramente definida e controlada, respeitando-se limites claros de qualidade.

Van Looy, De Backer e Poels, (2011) analisam os conceitos que diferenciam maturidade e níveis de maturidade, bem como capabilidade e níveis de capabilidade, do ponto de vista do CMMI e outros modelos. A maturidade indica até que ponto uma organização coloca em prática, de forma explícita e consistente, seu processo de negócio. Já capabilidade compreende as competências para se alcançar os objetivos estabelecidos.

Em (Proença e Borbinha, 2018) é demonstrada a capacidade de classificação de forma automática de níveis de capabilidade e maturidade com base em algumas características de processos de organizações. Para tal são utilizados o modelo CMMI em seus três componentes (Desenvolvimento, Serviços e Aquisição) e a norma ISO/IEC 15504-5 (SPICE - Software Process Improvement and Capability Evaluation)

\section{PESQUISA BIBLIOMÉTRICA}

Através de uma pesquisa bibliométrica na plataforma SCOPUS com as palavras-chaves “Industry 4.0" e "Maturity" entre os anos 2001 e 2018, observou-se um número crescente e significativo de publicações em periódicos, conforme Figura 2. Enquanto de 2001 a 2015 não se verificam publicações relacionadas ao tema, em 2016 surge um quantitativo de 13 trabalhos e em 2018 um total de 49 publicações. 


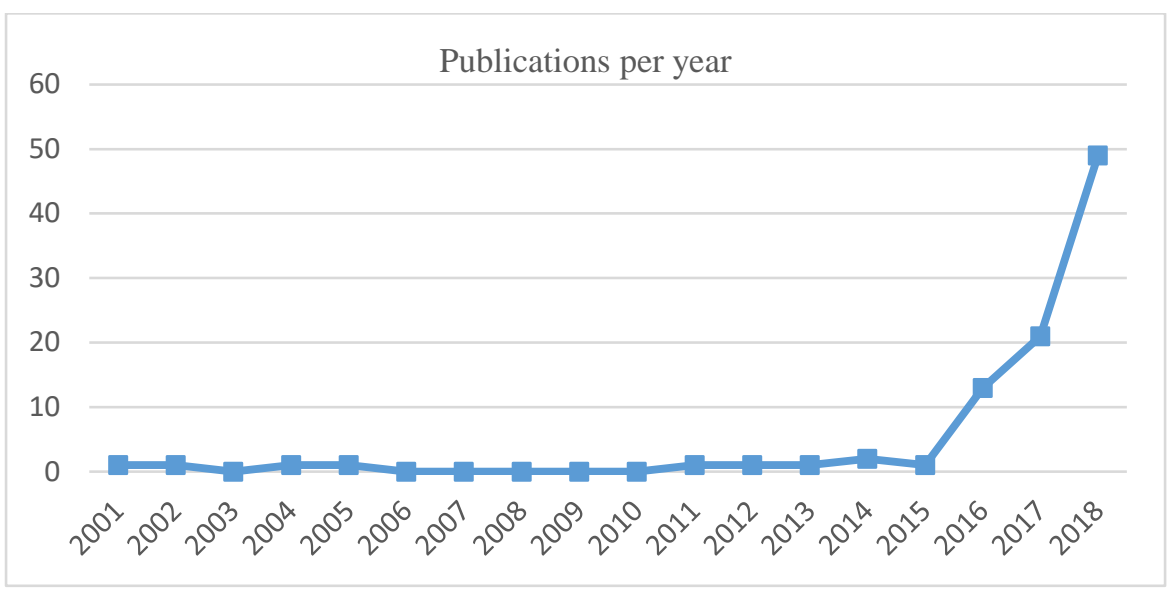

FIGURA 2. Número de publicações ao longo do tempo com as palavras-chave Industry 4.0 e Maturity. (SCOPUS)

Após realizada uma análise do material, foi possível destacar 7 modelos de maturidade para Indústria 4.0, além de outros dois modelos relevantes presentes na literatura (PwC, Acatech), conforme apresentado na Tabela 2.

TABELA 2. Modelos de Maturidade

\begin{tabular}{|l|l|l|l|l|}
\hline Autor & Ano & Nome & Dimensões organizacionais & $\begin{array}{l}\text { Níveis } \\
\text { Maturidade }\end{array}$ \\
\hline $\begin{array}{l}\text { Schumacher, } \\
\text { A., Erol, S., } \\
\text { Sihn, W }\end{array}$ & 2016 & & $\begin{array}{l}\text { 9: Estratégia, liderança, } \\
\text { clientes, produtos, } \\
\text { operações, cultura, pessoas, } \\
\text { governança e tecnologia }\end{array}$ & $\begin{array}{l}\text { 5 níveis (1=não } \\
\text { implementado a } \\
\text { 5=totalmente } \\
\text { implementado) }\end{array}$ \\
\hline $\begin{array}{l}\text { Jaione } \\
\text { Ganzarain } \\
\text { Nekane } \\
\text { Errasti }\end{array}$ & 2016 & & $\begin{array}{l}\text { 3 estágios: Envision 4.0; } \\
\text { enable 4.0; enact 4.0 }\end{array}$ & $\begin{array}{l}\text { 5 níveis: Inicial, } \\
\text { gerenciado, } \\
\text { definido, } \\
\text { transformação e } \\
\text { Modelo de Negócio } \\
\text { detalhado. }\end{array}$ \\
\hline $\begin{array}{l}\text { Anna De } \\
\text { Carolis, } \\
\begin{array}{l}\text { Marco } \\
\text { Macchi, Elisa } \\
\text { Negri, e } \\
\text { Sergio Terzi }\end{array}\end{array}$ & 2017 & DREAMY & $\begin{array}{l}\text { 4 dimensões: Processos, } \\
\text { Controle e monitoramento, } \\
\text { Tecnologia e Organização. }\end{array}$ & $\begin{array}{l}\text { 5 níveis: Inicial, } \\
\text { gerenciado, } \\
\text { definido, integrado e } \\
\text { interoperado, } \\
\text { orientado } \\
\text { digitalmente. }\end{array}$ \\
\hline $\begin{array}{l}\text { Christian } \\
\text { Leyh, Thomas } \\
\text { Schäffer, } \\
\text { Katja Bley, }\end{array}$ & 2016 & SIMMI 4.0 & $\begin{array}{l}\text { 4: Integração vertical, } \\
\text { horizontal, desenvolvimento } \\
\text { digital, cruzamento de } \\
\text { tecnologias. }\end{array}$ & $\begin{array}{l}\text { 5 estágios de } \\
\text { digitalização: } \\
\text { Básica, cruzada, } \\
\text { horizontal e vertical, }\end{array}$ \\
\hline
\end{tabular}




\begin{tabular}{|c|c|c|c|c|}
\hline $\begin{array}{l}\text { Sven } \\
\text { Forstenhäusler }\end{array}$ & & & & total. \\
\hline $\begin{array}{l}\text { Ebru Gökalp, } \\
\text { Umut Şener, } \\
\text { P. Erhan Eren }\end{array}$ & 2017 & & $\begin{array}{l}\text { 5: Gestão de ativos, } \\
\text { Governança de dados, } \\
\text { Gestão de aplicações, } \\
\text { transformação de processos, } \\
\text { alinhamento organizacional }\end{array}$ & $\begin{array}{l}\text { 6: incompleto, } \\
\text { realizado, } \\
\text { gerenciado, } \\
\text { estabilizado, } \\
\text { preditivo, otimizado. }\end{array}$ \\
\hline $\begin{array}{l}\text { Canetta, L; } \\
\text { Barni, A; } \\
\text { Montini, E }\end{array}$ & 2018 & & $\begin{array}{l}\text { 5: Estratégia, Processos, } \\
\text { Produtos e Serviços, } \\
\text { Tecnologias, Recursos } \\
\text { Humanos. }\end{array}$ & $\begin{array}{l}\text { 4: Ausência, } \\
\text { Iniciante, } \\
\text { Intermediário, } \\
\text { Experiente. }\end{array}$ \\
\hline $\begin{array}{l}\text { Sjodin, DR; } \\
\text { Parida, V; } \\
\text { Leksell, M; } \\
\text { Petrovic, A }\end{array}$ & 2018 & & $\begin{array}{l}\text { 3: Pessoas, Processos, } \\
\text { Tecnologias }\end{array}$ & $\begin{array}{l}\text { 4: Tecnologias } \\
\text { conectadas, Coleta e } \\
\text { compartilhamento } \\
\text { de dados } \\
\text { estruturados, Análise } \\
\text { e otimização de } \\
\text { processos em tempo } \\
\text { real; Manufatura } \\
\text { inteligente e } \\
\text { preditiva. }\end{array}$ \\
\hline $\begin{array}{l}\text { Geissbauer, } \\
\text { R., Vedso, J., } \\
\text { Schrauf, S }\end{array}$ & 2016 & $\mathrm{PwC}$ & $\begin{array}{l}\text { 7: negócios, produtos e } \\
\text { serviços, integração da } \\
\text { cadeia de valor, análise de } \\
\text { dados, arquitetura de TI ágil, } \\
\text { conformidade e segurança, } \\
\text { organização e cultura. }\end{array}$ & $\begin{array}{l}\text { 4: iniciante, } \\
\text { integrador vertical, } \\
\text { colaboração } \\
\text { horizontal, } \\
\text { especialista digital. }\end{array}$ \\
\hline $\begin{array}{l}\text { Schuh, G., } \\
\text { Anderl, R., } \\
\text { Gausemeier, } \\
\text { J., Hompel, } \\
\text { M., Wahlster, } \\
\text { W }\end{array}$ & 2017 & ACATECH & $\begin{array}{l}\text { 4: Recursos, Sistemas de } \\
\text { Informação, Cultura, } \\
\text { Estrutura Organizacional. }\end{array}$ & $\begin{array}{l}\text { 6: Informatização, } \\
\text { Conectividade, } \\
\text { Visibilidade, } \\
\text { Transparência, } \\
\text { Capacidade } \\
\text { preditiva, } \\
\text { Adaptabilidade. }\end{array}$ \\
\hline
\end{tabular}

Todos os modelos presentes na Tabela, buscam avaliar as organizações através de diferentes Dimensões Organizacionais, sendo que em cada dimensão é realizada sua avaliação de nível de maturidade, variando de 4 a 6 níveis.

Em Schumacher et al (2016), é desenvolvido um modelo baseado em 62 itens de maturidade em aspectos de tecnologia e de organização, agrupados em nove dimensões de uma 
empresa, avaliando-se cada uma dentro de cinco níveis de maturidade. O modelo foi então aplicado em alguns estudos de casos.

Ganzarain e Errasti (2016) propõe um modelo de processo como uma ferramenta orientada para a visão, estratégia e construção de ações. Além disso, é definida uma escala de 5 níveis para cada modelo.

No estudo denominado DREAMY (De Carolis et al, 2017), inicialmente 5 grandes áreas são estruturadas: Projeto e Engenharia, Gestão da Produção, Gestão da Qualidade, Gestão da Manutenção e Gestão da Logística. Com isso 5 níveis de maturidade são definidos: Inicial, gerenciado, definido, integrado e interoperado e orientado digitalmente. Para se avaliar a capacidade digital de uma organização, não são consideradas apenas as tecnologias utilizadas. Com isso é avaliada a prontidão digital de uma companhia através de quatro dimensões: Processos, Controle e Monitoramento, Tecnologia e Organização.

O Modelo de Maturidade para Integração de Sistemas (SIMMI 4.0) (Leyh et al, 2016) permite às empresas classificar seus próprios sistemas de TI de acordo com o panorama da Indústria 4.0. Consiste de cinco estágios de maturidade, no qual cada um representa um nível correspondente de maturidade para Industria 4.0. Adicionalmente, cada estágio é dividido em quatro dimensões que representam diferentes componentes da empresa (Vertical, Horizontal, Desenvolvimento Digital e Cruzamento de tecnologias).

Em (Gökalp et al, 2017) é apresentado um modelo de maturidade para Indústria 4.0 baseada na norma ISO/IEC 15504, também conhecida como SPICE (Software Process Improvement and Capability Determination). O modelo é construído sob cinco aspectos ou dimensões e seis níveis de maturidade.

Canetta, Barni e Montini, (2018) desenvolveram um modelo baseado em 36 questões divididas em cinco sessões: Estratégia, Processos, Produtos e Serviços, Tecnologia e Recursos Humanos. Cada questão é então dividida em quatro diferentes níveis de maturidade: Ausência, Iniciante, Intermediário, Experiente. Com o resultado é gerado então um gráfico do tipo radar e uma média do nível geral de maturidade da companhia, com base nas cinco dimensões.

Em (Sjödin, 2018), cinco estudos de casos são analisados e os desafios para e os passos chave necessários para se implementar uma fábrica inteligente. É então construído um modelo com base em três princípios: Pessoas, introdução de processos ágeis e configuração de 
tecnologias modulares. Estas dimensões são então classificadas em quatro níveis de maturidade. Além disso são analisados os benefícios ao final da implementação de uma fábrica inteligente: maior eficiência dos processos, menor custo operacional, maior qualidade dos produtos e maior segurança e sustentabilidade.

O Modelo da PwC (Geissbauer, Vedso e Schrauf, 2016) é baseado em sete dimensões de capacidades digitais, envolvendo negócios, produtos e serviços, integração da cadeia de valor, análise de dados, arquitetura ágil de TI, conformidade e segurança, organização e cultura. Além disso quatro níveis de maturidade são apresentados: iniciante, integrador vertical, colaborador horizontal e especialista digital.

\subsection{Modelo Acatech}

Elaborado pela Academia Nacional de Ciências e Engenharia da Alemanha (ACATECH) no ano de 2017, o modelo de maturidade da Indústria 4.0 (Schuh et al, 2017) pode ser considerado além de um modelo de avaliação da maturidade em si, mas também um guia para que as organizações compreendam o caminho que devem seguir para o desenvolvimento da I 4.0. Apesar das tecnologias avançadas tornarem possível grandes possibilidades, a habilidade de desenvolver o potencial de transformação desses dados depende de cada organização, de acordo com sua cultura e estrutura organizacional.

O guia define quatro áreas estruturais: Recursos, Sistemas de Informação, Estrutura Organizacional e Cultura Organizacional. Cada área possui dois princípios fundamentais de acordo com a Tabela 3:

TABELA 3. Áreas estruturais e princípios fundamentais - Acatech (Schuh et al, 2017)

\begin{tabular}{|l|l|}
\hline \multicolumn{1}{|c|}{ Área Estrutural } & \multicolumn{1}{|c|}{ Princípio Fundamental } \\
\hline \multirow{2}{*}{ Recursos } & Capacidade Digital \\
\cline { 2 - 2 } & Comunicação Estruturada \\
\hline Sistemas de Informação & $\begin{array}{l}\text { Auto aprendizado no processamento da } \\
\text { informação }\end{array}$ \\
\hline
\end{tabular}




\begin{tabular}{|c|c|}
\hline & Integração dos Sistemas de Informação \\
\hline \multirow[t]{2}{*}{ Estrutura Organizacional } & Organização Interna Orgânica \\
\hline & $\begin{array}{l}\text { Colaboração Dinâmica ao longo da cadeia de } \\
\text { valor }\end{array}$ \\
\hline \multirow[t]{2}{*}{ Cultura Organizacional } & Abertura para mudança \\
\hline & Colaboração Social \\
\hline
\end{tabular}

Um dos princípios da Indústria 4.0 é a capacidade de mudança e adaptação de uma organização. No modelo da Acatech, são apresentados 6 níveis de maturidade, refletidos nas quatro áreas estruturais. Para que as transformações esperadas na empresa sejam realizadas de forma holística, um determinado nível de maturidade só é atingido quando todas as quatro perspectivas atingirem esse nível.

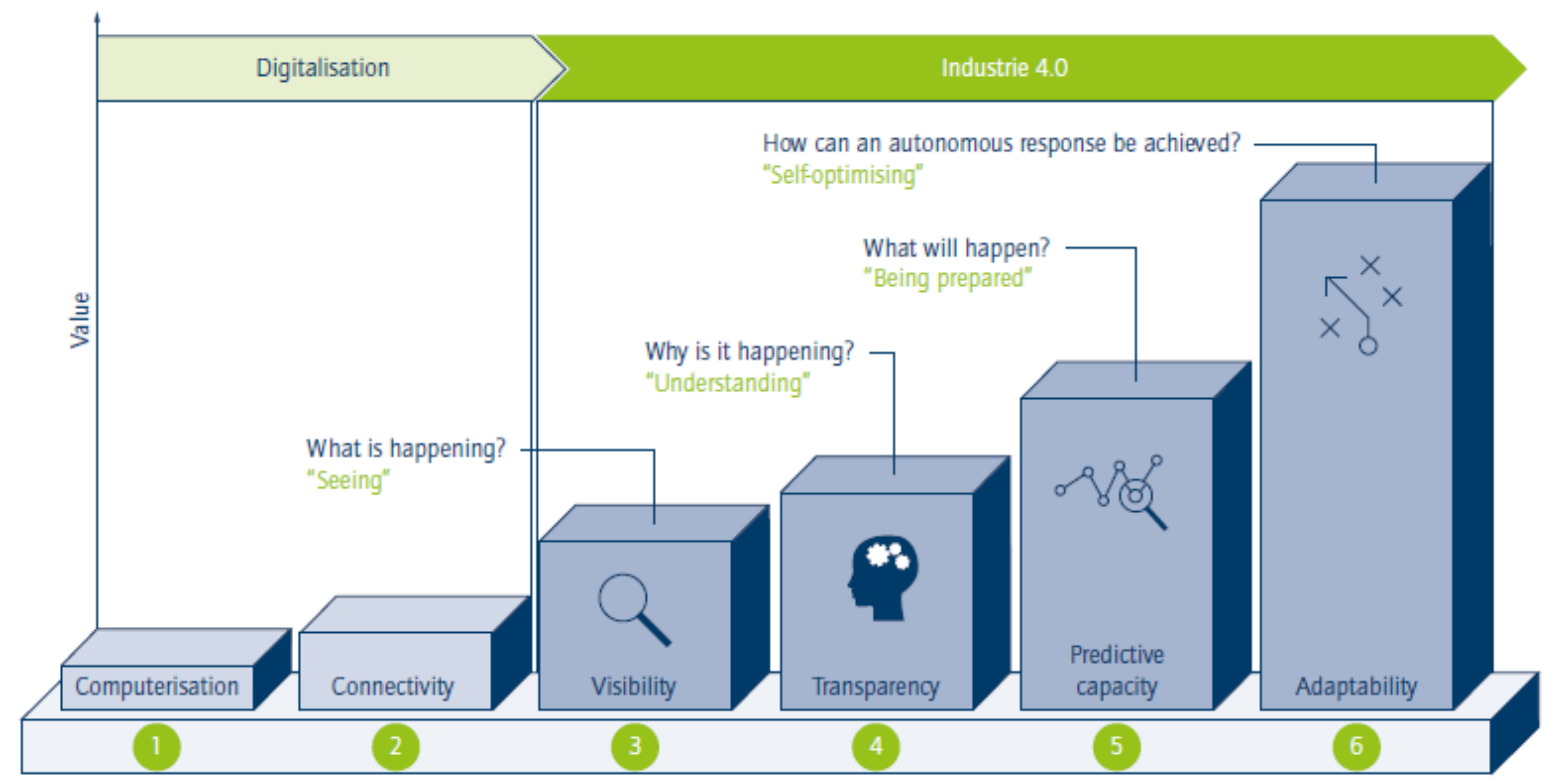

FIGURA 3. Níveis de maturidade para Indústria 4.0 (Schuh et al, 2017) O modelo apresenta seis níveis para o caminho para a Indústria 4.0

- Informatização: Fornece a base para a digitalização, sendo utilizada para a execução de tarefas repetitivas de maneira mais eficiente. Máquinas CNC que ainda não estão integradas a sistemas CAD são exemplos deste nível. 
- Conectividade: Os sistemas de negócio são atualizados ou substituídos por sistemas conectados e interoperados, porém a integração completa dos sistemas de informação e sistemas de operação ainda não está implantada.

- Visibilidade: Obtido através do sensoriamento e aquisição de dados. Possibilita que os gerentes tomem decisões baseadas em dados da produção, disponibilizados para os envolvidos ao longo da cadeia de valor.

- Transparência: Compreende a interpretação dos dados obtidos no nível anterior. Busca a geração do conhecimento, a identificação de problemas e a criação de padrões de ações visando a resolução rápida de falhas. Estão presentes ferramentas de Mineração de Dados e softwares de simulação e análise probabilística.

- Capacidade Preditiva: A organização é capaz de simular diferentes cenários, automatizando processos como a antecipação de paradas de produção, atrasos, variações de demanda, dentre outros. São utilizadas ferramentas de Inteligência Artificial como Raciocínio Baseado em Caso.

- Adaptabilidade: A adaptação contínua permite à organização delegar as ações a sistemas de informação e comunicação em conjunto com sistemas de operação, obtendo maior velocidade de resposta a situações de mudança. A adaptabilidade ocorre quando a empresa é capaz de usar dados do modelo digital (Sistemas Ciber Físicos) para tomar decisões que possibilitem os melhores resultados em menor tempo, de forma automática.

\section{CONCLUSÃO}

Um estudo foi realizado com o objetivo de apresentar diversos modelos de maturidade e capabilidade, desde o CMM, desenvolvido nos anos 80 e os modelos presentes na literatura atual para Indústria 4.0, com destaque para o modelo da Acatech.

Modelos de maturidade podem ser definidos como estruturas conceituais, constituído por partes que definem o estado de desenvolvimento de uma determinada área de interesse, descrevendo processos que uma organização precisa desenvolver para atingir um determinado cenário futuro desejado. São baseados nas premissas de que as pessoas, as organizações, áreas funcionais, e os processos, evoluem através de um processo de desenvolvimento, na direção de uma maturidade mais avançada. $\mathrm{O}$ objetivo dos modelos de maturidade é quantificar as atividades realizadas, torná-las mensuráveis e fazê-las maduras ao longo do tempo. 


\section{REFERÊNCIAS}

CANETTA, L., BARNI, A., \& MONTINI, E. (2018). "Development of a Digitalization Maturity Model for the manufacturing sector". In 2018 IEEE International Conference on Engineering, Technology and Innovation (ICE/ITMC), IEEE, pp. 1-7.

DE CAROLIS, A., MACCHI, M., NEGRI, E., \& TERZI, S. (2017). “A maturity model for assessing the digital readiness of manufacturing companies". In IFIP International Conference on Advances in Production Management Systems, Springer, Cham., pp. 13-20

DRATH, R., \& HORCH, A. (2014). Industrie 4.0: Hit or hype?[industry forum]. IEEE industrial electronics magazine, 8(2), 56-58.

GANZARAIN, J., \& ERRASTI, N. (2016). “Three stage maturity model in SME's toward industry 4.0”. Journal of Industrial Engineering and Management (JIEM),Vol. 9, No. 5, 1119-1128.

GEISSBAUER, R., VEDSO, J., \& SCHRAUF, S. (2016). "Industry 4.0: Building the digital enterprise”. Retrieved from PwC Website: https://www. pwc. com/gx/en/industries/industries-4.0/landing-page/industry-4.0-buildingyour-digital-enterprise-april-2016. Pdf [Accessed: 06-Mar-2019].

GÖKALP, E., ŞENER, U., \& EREN, P. E. (2017). "Development of an assessment model for industry 4.0: industry 4.0-MM”. In International Conference on Software Process Improvement and Capability Determination, Springer, Cham., pp. 128-142

GORECKY, D., SCHMITT, M., LOSKYLL, M., \& ZÜHLKE, D. (2014). Human-machine-interaction in the industry 4.0 era. In 2014 12th IEEE International Conference on Industrial Informatics (INDIN) (pp. 289-294).

HELGESSON, Y. Y. L., HÖST, M., \& WEYNS, K. (2012). A review of methods for evaluation of maturity models for process improvement. Journal of Software: Evolution and Process, 24(4), 436-454.

JAZDI, N. (2014). Cyber physical systems in the context of Industry 4.0. In 2014 IEEE international conference on automation, quality and testing, robotics (pp. 1-4). IEEE.

MELO, M. O., CAVALCANTI, G. A., GONÇALVES, H. S., \& DUARTE, S. T. (2007). Inovações Tecnológicas na Cadeia Produtiva Têxtil: análise e estudo de caso em indústria no nordeste do Brasil. Revista Produção Online, 7(2).

LASI, H., FETTKE, P., KEMPER, H. G., FELD, T., \& HOFFMANN, M. (2014). Industry 4.0. Business \& information systems engineering, 6(4), 239-242.

LEE, J., BAGHERI, B., \& KAO, H. A. (2015). A cyber-physical systems architecture for industry 4.0-based manufacturing systems. Manufacturing letters, 3, 18-23.

LEYH, C., BLEY, K., SCHÄFFER, T., \& FORSTENHÄUSLER, S. (2016). "SIMMI 4.0-a maturity model for classifying the enterprise-wide it and software landscape focusing on Industry 4.0". In 2016 Federated Conference on Computer Science and Information Systems (FedCSIS), IEEE, pp. 1297-1302

LI, Z., WANG, Y., \& WANG, K. S. (2017). Intelligent predictive maintenance for fault diagnosis and prognosis in machine centers: Industry 4.0 scenario. Advances in Manufacturing, 5(4), 377-387.

PAULK, M. C. (2009). A history of the capability maturity model for software. ASQ Software Quality Professional, 12(1), 5-19.

PROENÇA, D., \& BORBINHA, J. (2018). Formalizing ISO/IEC 15504-5 and SEI CMMI v1. 3-Enabling automatic inference of maturity and capability levels. Computer Standards \& Interfaces, 60, 13-25.

SCHUH, G., ANDERL, R., GAUSEMEIER, J., HOMPE, M. T., \& WAHLSTER, W. (2017). Industrie 4.0 Maturity Index: Managing the Digital Transformation of Companies; acatech STUDY: Munich.

SCHUMACHER, A., EROL, S., \& SIHN, W. (2016), “A maturity model for assessing industry 4.0 readiness and maturity of manufacturing enterprises”, Procedia CIRP, Vol. 52, pp. 161-166.

SJÖDIN, D. R., PARIDA, V., LEKSELL, M., \& PETROVIC, A. (2018). "Smart Factory Implementation and Process Innovation: A Preliminary Maturity Model for Leveraging Digitalization in Manufacturing Moving to 
smart factories presents specific challenges that can be addressed through a structured approach focused on people, processes, and technologies". Research-Technology Management, Vol. 61, No. 5, 22-31.

SPENDLA, L., KEBISEK, M., TANUSKA, P., \& HRCKA, L. (2017). Concept of predictive maintenance of production systems in accordance with industry 4.0. In 2017 IEEE 15th International Symposium on Applied Machine Intelligence and Informatics (SAMI) (pp. 000405-000410). IEEE.

TEAM, C. P. (CMMI)(2002). Capability maturity model® integration (CMMI SM), version 1.1. CMMI for Systems Engineering, Software Engineering, Integrated Product and Process Development, and Supplier Sourcing (CMMI-SE/SW/IPPD/SS, V1. 1).

VAN LOOY, A., DE BACKER, M., \& POELS, G. (2011). Defining business process maturity. A journey towards excellence. Total Quality Management \& Business Excellence, 22(11), 1119-1137.

WANG, K. S. (2014). Intelligent and integrated RFID (II-RFID) system for improving traceability in manufacturing. Advances in Manufacturing, 2(2), 106-120. 